\title{
Cognitive Chaotic UWB-MIMO Detect-Avoid Radar for Autonomous UAV Navigation
}

\author{
Yogesh Nijsure ${ }^{\dagger}$, Member, IEEE, Georges Kaddoum, Member, IEEE, Nazih Khaddaj Mallat, Senior \\ Member, IEEE, Ghyslain Gagnon, Member, IEEE, Francois Gagnon, Senior Member, IEEE \\ $\dagger$ Corresponding author, e-mail: y.nijsure.2014@ieee.org
}

\begin{abstract}
A cognitive detect and avoid radar system based on chaotic UWB-MIMO waveform design to enable autonomous UAV navigation is presented. A Dirichlet-Process-Mixture-Model (DPMM) based Bayesian clustering approach to discriminate extended targets and a Change-Point (CP) detection algorithm are applied for the autonomous tracking and identification of potential collision threats. A DPMM based clustering mechanism does not rely upon any a priori target scene assumptions and facilitates online multivariate data clustering/classification for an arbitrary number of targets. Furthermore, this radar system utilizes a cognitive mechanism to select efficient $c$ haotic waveforms to facilitate enhanced target detection and discrimination. We formulate the CP mechanism for the online tracking of target trajectories which present a collision threat to the UAV navigation and thus we supplement the conventional Kalman filter based tracking. Simulation results demonstrate a significant performance improvement for the DPMM-CP assisted detection as compared with direct generalized likelihood ratio based detection. Specifically, w e observe a $4 \mathrm{~d} B$ p erformance gain in target detection over conventional fixed $U$ WB $w$ aveforms and superior collision avoidance capability offered by the joint DPMM-CP mechanism.
\end{abstract}

Index Terms-Cognitive Radar, Chaotic UWB radar waveform, Dirichlet-Process-Mixture-Model based discrimination, Change-Point detection, Autonomous UAV Navigation.

\section{INTRODUCTION}

Unmanned Aerial Systems (UAS) have gained a tremendous importance during recent years in civilian and military applications alike. These applications typically monitor the phenomenon of interest in real-time and relay the corresponding data to a central platform to allow an effective and timely response [1], [2]. Surveillance systems are being used for both military and civilian operations [3]-[6] and, therefore, it is imperative to design these systems for different deployment scenarios and conditions. More recently in early 2015, the Federal Aviation Administration (FAA) released its much anticipated regulations for the use of unmanned aircraft or Unmanned Aerial Vehicle (UAV) drones for commercial purposes in domestic airspace [7]. A critical design

Copyright (c) 2016 IEEE. Personal use of this material is permitted. However, permission to use this material for any other purposes must be obtained from the IEEE by sending a request to pubs-permissions@ieee.org.

Yogesh Nijsure, Georges Kaddoum, Ghyslain Gagnon, and Francois Gagnon are with University of Québec, ETS, 1100 Notre-Dame west, H3C 1K3, Montreal, Canada (e-mail: y.nijsure.2014@ieee.org; georges.kaddoum@etsmtl.ca; Ghyslain.Gagnon@etsmtl.ca; Francois.Gagnon@etsmtl.ca). Nazih Khaddaj Mallat is with Al Ain University of Science and Technology (AAU), P.O. Box 64141 Al Ain, United Arab Emirates (UAE) (email: nazih.mallat@aau.ac.ae). problem in existing UAV navigation capacity is the ability to autonomously detect/sense and avoid collisions with other UAV drones operating in close proximity [7]-[11]. The critical requirements to allow an autonomous UAV navigation are based on assurance on inter-UAV separation, long range (time to collision $>30 \mathrm{sec}$ ) and short range (time to collision $<30 \mathrm{sec}$ ) collision avoidance mechanisms [11].

Several collision avoidance mechanisms including Automatic Dependant Surveillance Broadcast (ADS-B) and Traffic Collision Avoidance System (TCAS) have been proposed to report the real-time GPS location of the UAVs [12]-[14]. Since these mechanisms rely upon open and un-encrypted transmission signals, they are invariably prone to spoofing and other message infringement forms of attacks [15]. Other approaches include, segregated or designated airspace for UAS operations, traditional visual see and avoid based on optical sensors [8]-[10], cooperative separation assurance strategy that could be based on a communications link between multiple UAV systems, and ground based radar surveillance [11]. All of these approaches inhibit the ability of the UAV drone to be fully autonomous in terms of decision making to implement collision avoidance maneuvers.

In this work, we envision a fully autonomous UAV navigation scheme facilitated by 'Detect and Avoid' (DAA) on-board radar implementation. Specifically, we utilize an Electronically Scanned Array (ESA) based Ultra-Wideband (UWB) collocated Multiple Input Multiple Output (MIMO) radar to implement our novel autonomous collision avoidance strategy. This proposed strategy benefits from the key concept of radar cognition, which imparts to the radar an ability to dynamically adapt the UWB-MIMO radar transmission waveform to enhance the UAV target detection. Consequently, this cognition facilitates better estimation of imminent collision points, in order to assist the UAV guidance and navigation.

From an hardware design perspective, our approach utilizes the cognitive monostatic UWB-MIMO radar coupled with the usage of chaos based UWB waveforms which offer tremendous flexibility in the design of key radar transmission parameters which include the UWB monocycle pulsewidth, Pulse Repetition Interval (PRI) and UWB monocycles phase/amplitude. Specifically, the chaotic UWB-MIMO radar design supports significantly large degrees of freedom in choosing transmission waveform with chaotic amplitude, phase and PRI, thus imparting higher degree of freedom within waveform design and selection. As a result, chaotic UWB waveforms exhibit pronounced sensitivity to scattering relative 


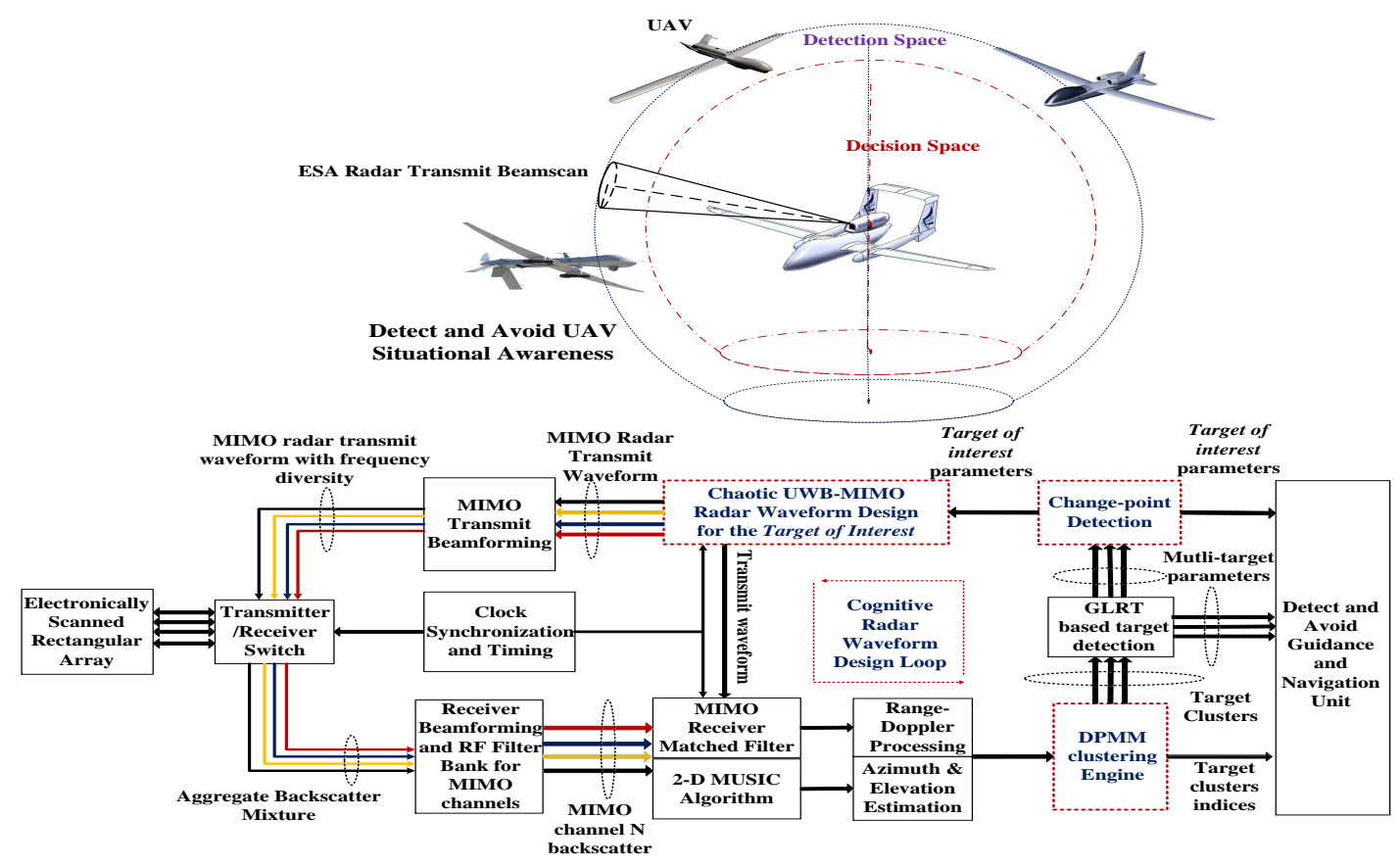

Fig. 1. System architecture.

to conventional radar signals, as shown in works [16]-[18].

From an algorithmic perspective, in order to discriminate between distinct extended targets, there is a need to develop a robust clustering algorithm that will classify and attribute the received signal contributions to each individual target. Most clustering or discrimination algorithms, $K$ means clustering [19] needs to make a priori assumptions about the number of targets present in the environment. The number of scattering centers and the number of corresponding extended targets are in general unknown a priori, and are to be inferred directly from the backscatter data. Thus, there is a need to utilize an unsupervised mixture component analysis technique, which can offer unbounded complexity and can be used to effectively discriminate between extended target signatures. One such effective mechanism is the Bayesian nonparametric technique for discrimination. The Bayesian nonparametric approach has been adopted in various applications, including target tracking [20], and high dimensional data clustering [21], [22]. Moreover, this technique has also been applied to cluster identification in Synthetic Aperture Radar (SAR) images [23]. More recently, it was also utilized in clustering of chaotic UWB backscatter signals for a bistatic UWB-MIMO cognitive radar setup [17], [24]. In this work, we utilize a robust nonparametric Bayesian clustering based algorithm, called the Dirichlet-Process-Mixture-Model (DPMM) as shown in works [17], [20], [21], [25].

In addition to the DPMM based clustering mechanism we also adopt a Change-Point (CP) detection algorithm to allow the UAV to autonomously monitor and determine imminent collision with other UAV targets in its proximity. Specifically this $\mathrm{CP}$ algorithm is based on online Bayesian estimation of change-points in the estimated UAV tracks corresponding to UAV targets in the proximity. Our objective is to determine the sudden change points within the estimated trajectories of the surrounding UAV targets and to quickly identify the the imminent collisions, so that the guidance and navigation unit can make coarse correction to its own trajectory. Details on the $\mathrm{CP}$ algorithm based on perfect simulation approach can be found in works like [26]-[28].

\section{A. Motivation for the proposed research}

The proposed cognitive chaotic UWB-MIMO radar is designed to impart the UAV with complete autonomy with respect to decision making, specifically in terms of executing course-correction maneuvers in order to avoid imminent collisions. The key motivation behind the proposed system design is to integrate fully autonomous data driven statistical mechanisms which can support a cognitive radar architecture, which could enable, (i) cognitive waveform selection/design to enhance target detection, (ii) Unsupervised mixture component analysis capability offered by DPMM approach which is fully raw-data driven and does not need any a priori radar scene assumptions, and (iii) $\mathrm{CP}$ algorithm which enables online trajectory change point estimation to facilitate collision avoidance with respect to sudden changes over the trajectory for the target of interest. In summary, the proposed cognitive Bayesian DPMM-CP framework provides significant advantage over conventional radar approaches [29]-[31] for UAVs, due to it's ability to address autonomous target discrimination, imminent collision threat detection for executing course-correction maneuvers and a cognitive waveform design architecture to facilitate enhanced target detection and tracking.

\section{B. Key Innovation and Advantages}

The proposed cognitive Bayesian DPMM-CP radar framework offers several advantages over existing approaches which 
facilitate sense and avoid solutions. Some of the key innovations and advantages of the proposed approach include

- Ability to function at all times during the day and in all weather conditions unlike optical sensor based solutions [8]-[10].

- Significant resilience to interception and spoofing attempts in comparison to ADS-B and TCAS based solutions.

- Communication based sense and avoid mechanisms like ADS-B and TCAS have an inherent dependency on the transponder of the target UAV. These mechanisms suffer if the target is hostile or non-cooperative or if it is unequipped with a transponder [31], [32]. Other issues include response time latency which could render TCAS based systems of little or no use [33], failure to detect anomalous situations including altitude-reporting errors because of intruders that are maneuvering in a manner incompatible with the TCAS-Resolution Advisory (RA). A detailed discussion on the shortcomings of TCAS based systems for application to UAVs is presented in [31][33]. Moreover, works like [33], propose the usage of on-board radar based systems to alleviate these mentioned problems associated with ADS-B and TCAS based sense and avoid mechanisms.

- Enhanced target detection compared to fixed UWBMIMO conventional radar waveforms due to chaotic variation in transmission parameters.

- DPMM based target clustering mechanism which does not require any a priori target scene assumptions and can operate on raw data to discriminate multiple UAV target signatures.

- Online trajectory changes estimation facilitated by $\mathrm{CP}$ algorithm to isolate and monitor the target of interest which helps with the execution of collision avoidance maneuvers.

Major contributions for this work can be summarized as follows:

1) Development of a cognitive radar mechanism to enable the adaptation of the chaotic UWB-MIMO waveform parameters with an objective of enhancing the target of interest signatures within the radar backscatter.

2) Development of a robust DPMM clustering framework for extended target detection and discrimination of the multiple UAV targets.

3) Usage of $\mathrm{CP}$ algorithm based on perfect simulation to estimate the sudden variation in trajectories of the UAV targets to avoid imminent collisions.

The rest of the paper is organized as follows: in Section II, we provide a general overview of the proposed cognitive system architecture. In Section III, we present the actual DPMM clustering for the backscatter from the extended UAV targets scenario. Section IV presents the CP algorithm based on perfect simulation to enable the proposed autonomous DAA strategy. Simulation results are described in detail in Section V. Finally, in Section VI, we provide concluding remarks and potential applications. Throughout this work, we use $(\cdot)^{T}$ to denote matrix transpose. We use $\mathcal{N}(\mu, \sigma)$ to denote the (multivariate) Gaussian distribution with mean vector $\mu$ and covariance matrix $\sigma$.

\section{System Architecture of the Proposed Cognitive RADAR DESIGN}

A general system architecture for the distributed MIMO radar system is shown in Fig. 1. The transmission waveform orthogonality is achieved through frequency diversity for the MIMO architecture that is shown. It is also assumed that the receiver has full knowledge of the transmitted waveform. We use an ensemble of chaos based UWB waveforms as shown in [16]. The UWB waveform ensemble consists of individual chaos based UWB waveforms in which the PRI, amplitude and phase are dictated by uniformly distributed random variables. Each normalized second derivative Gaussian UWB waveform can be represented as

$$
\begin{array}{r}
u(t)=\sum_{k=1}^{K} \gamma_{k}\left[1-4 \pi\left(\frac{t-\varphi_{k} T}{T_{p}}\right)^{2}\right] \\
\exp \left\{-2 \pi\left(\frac{t-\varphi_{k} T}{T_{p}}\right)^{2}\right\} \cos \left(\xi_{k}\right),
\end{array}
$$

where $K$ is the number of second derivative Gaussian monocycles within the UWB waveform, $T_{p}$ is the pulsewidth of the single UWB pulse, $\gamma_{k}$ represents the normalized amplitude of the $k^{t h}$ monocycle, which is uniformly distributed, $\varphi_{k} T$ is the uniformly distributed random pulse repetition time between $[0, T], \xi_{k}$ represents the phase of the $k^{t h}$ pulse. The phase $\xi_{k}$ is chosen as 0 or $\pi$ in accordance with a pseudo-random binary sequence.

\section{A. Signal Model}

Consider a mono-static MIMO radar system with $M_{c}$ and $M_{r}$ antenna elements which represent the columns and rows within the Uniform Rectangular Array (URA) respectively. As shown in Fig. 1, we consider a monostatic radar case, hence the same rectangular array is utilized with a transmit/receive switch within the radar system design. Let $N_{r}$ represent an arbitrary structure receiver array that could be selected while receiving the backscatter signal. We adopt the MIMO URA architecture as shown in [34].

Let $\mathbf{u}(t)=\left[u_{1}(t), \cdots, u_{N}(t)\right]$, be the $N \times 1$ vector of orthogonal UWB-MIMO chaotic waveforms, which satisfies the orthogonality condition $\int_{T_{p}} \mathbf{u}(t) \mathbf{u}^{H}(t)=\mathbb{I}_{N}$, where $\mathbb{I}_{N}$ represents the identity matrix of size $N$. The variable notation $N$ signifies the distinct MIMO channels or in other words the distinct beams designed with the 2D planar array. In our work, we assume that the orthogonality between $N$ distinct MIMO channels is assumed over the frequency domain, which implies that each UWB signal within $\mathbf{u}(t)$ has a distinct center frequency of operation.

Assuming $\kappa$ number of target centers which belong to the several range-Doppler bins and are illuminated within a particular 2D scan of the planar array, the $N_{r} \times 1$ receiver array signal vector, and can be represented as, 


$$
\begin{array}{r}
\mathbf{r}(\tau, t)=\sum_{i=1}^{\kappa}\left[\zeta_{i}\left(\theta_{i}, \phi_{i}, \tau\right) \beta\left(\theta_{i}, \phi_{i}\right)\right. \\
\left.\left(\mathbf{W}^{H} \alpha\left(\theta_{i}, \phi_{i}\right)\right)^{H} \mathbf{u}(t)\right]+\eta(t, \tau)
\end{array}
$$

where $t$ and $\tau$ are the fast and slow time indices, respectively. $\mathbf{W}=\left[w_{1}, \cdots, w_{N}\right]$ is the $M_{c} M_{r} \times N$ transmit beamforming matrix, and $(\cdot)^{H}$ represents the Hermitian transpose. $\beta\left(\theta_{i}, \phi_{i}\right)$ represents the $N_{r} \times 1$ beam steering vector for the chosen receiver array. $\zeta_{i}\left(\theta_{i}, \phi_{i}, \tau\right)$ represents the reflection coefficient of the target center located at $\left\{\theta_{i}, \phi_{i}\right\}$ with a variance of $\sigma_{\zeta}^{2}$, and $\eta(\tau, t)$ represents the zero mean white Gaussian noise with variance $\sigma_{\eta}^{2} \cdot \alpha(\theta, \phi)=\operatorname{vec}\left(\mathbf{U} \odot \mathbf{a}(\theta, \phi) \mathbf{b}^{T}(\theta, \phi)\right)$, where $\mathbf{U}$ represents a $M_{c} \times M_{r}$ matrix of ones representing the presence of the elements in $\{\mathrm{c}, \mathrm{r}\}$ location within the $2 \mathrm{D}$ array. $\alpha(\theta, \phi)$ is an $M_{c} M_{r} \times 1$ beam steering vector for azimuth angle $\phi$ and elevation angle $\theta$. vec(.) stands for the operator which stacks columns of the matrix into a single column vector. $\odot$ represents the Hadamard product. $\mathbf{a}$ and $\mathbf{b}$ are vectors of $M_{c} \times$ 1 and $M_{r} \times 1$ dimensions respectively as defined in [34]. We assume a Swerling II target model which implies that the target reflection coefficient remains constant within the duration of the radar pulse but varies from pulse to pulse.

The matched filtered output of the received signal $\mathbf{r}(\tau, t)$ can be represented as [34],

$$
\begin{aligned}
\mathbf{s}_{n}(\tau) & =\int_{T} \mathbf{r}(t, \tau) u_{n}^{*}(t) d t \\
& =\sum_{i=1}^{\kappa}\left[\zeta_{i}\left(\theta_{i}, \phi_{i}, \tau\right)\left(w_{n}^{H} \alpha\left(\theta_{i}, \phi_{i}\right)\right)^{*}\right. \\
& \left.\times \beta\left(\theta_{i}, \phi_{i}\right)\right]+\eta_{n}(\tau)
\end{aligned}
$$

where $(\cdot)^{*}$ stands for conjugation, $n=1, \cdots, N$, and $\eta_{n}=$ $\int_{T} \eta(t, \tau) u_{n}^{*}(t) d t$ is the $N_{r} \times 1$ noise term with covariance $\sigma_{\eta}^{2} \mathbb{I}_{N_{r}}$. We utilize the $2 \mathrm{D}$ transmit and receive beamforming mechanism, as shown in [34], which enables us to determine the optimal values of the weights $\mathbf{W}$ for the beam steering vectors, $\alpha(\theta, \phi)$ and $\beta(\theta, \phi)$. Also note that the extended targets occupying several range-Doppler bins have been modelled as a collection of Swerling II type targets and multiple $\zeta\left(\theta_{i}, \phi_{i}\right)$ target scatterer locations.

\section{B. Proposed Cognitive Bayesian DPMM-CP mechanism}

As shown in Fig. 1, the chaotic UWB-MIMO waveform $\mathbf{u}(t)$ is transmitted by the 2D planar array after the computation of the optimal beamforming weights $\mathbf{W}$ as shown in [34]. This monostatic UWB-MIMO radar system initially illuminates the entire elevation angular space $\Theta \in\left[0^{\circ}, 180^{\circ}\right]$ and the azimuth angular space $\Phi \in\left[-180^{\circ}, 180^{\circ}\right]$. Upon this illumination, the receiver array on the monostatic radar is enabled and the angular space $\{\Theta, \Phi\}$ is scanned. This receiver array scanning is enabled by the beamsteering matrix $\beta\left(\theta_{i}, \phi_{i}\right)$ and the receiver scanning is repeated for a predetermined duration to collect the target backscatter echo signals. Subsequently, the aggregate backscatter signal is filtered by the UWB-RF front-end to isolate the $N$ channel MIMO contributions over the $N_{r} \times 1$ receiver array. These $N$ channel contributions are recorded for future processing.

For a particular MIMO channel $n$, the corresponding matched filter response $\mathbf{s}_{n}$ is computed by evaluating (3). This channel backscatter signal is then operated by the well known 2D Multiple Signal Classification (MUSIC) Algorithm, to evaluate the angle and azimuth vector estimates for the backscatter signal over channel $n$. The matched filtering operation also generates the range-Doppler estimates for the received backscatter. These azimuth, elevation and rangeDoppler estimates are forwarded to the proposed DPMM clustering engine in order to cluster the backscatter signal over channel $n$.

The DPMM clustering algorithm generates the distinct clusters by evaluating the underlying $3 \mathrm{D}$ multivariate distribution over the received signal amplitude, azimuth and elevation angle estimates, $\{\Gamma, \phi, \theta\}$ for $\kappa$ target centers within the radar environment. For each discriminated cluster, a Generalized Likelihood Ratio Test (GLRT) is adopted to detect the presence of the target in a particular range-Doppler bin. The detected target clusters information is then passed on to the $\mathrm{CP}$ algorithm for enabling the KF track of each target and detecting the sudden change points in the trajectory of the target. The location estimate for the target within the closest proximity of the UAV is designated as the target of interest and this location estimate is relayed to the UWB-MIMO chaotic waveform design unit for determination of the optimal $T$, and $T_{p}$ for channel $n$. An optimal choice of $T_{p}$ and $T$ allows enhanced range and Doppler resolution for the target of interest.

This procedure is repeated for the entire set of backscattered signals over $N$ MIMO beams or channels, and an optimal MIMO waveform $\mathbf{u}(t)$ is designed for transmission in the next instant. The discriminated cluster parameters (output of DPMM block), the detected multi-target parameters (GLRT block output) and the target of interest location parameters (output of CP block), are relayed to the UAV guidance and navigation unit to make a decision on course correction and collision avoidance.

The red dashed boxes within Fig. 1 represent the novelty and major contributions brought by the proposed approach which allows the radar to autonomously detect neighbouring UAVs through application of DPMM based target clustering and imminent collision threats detection by $\mathrm{CP}$ algorithm operating on the close proximity targets within the decision space. The cognitive waveform design is thus facilitated by DPMM-CP mechanism which results in an optimal selection of $T$ and $T_{p}$ for each channel and for each instance of radar interrogation or transmission. The motivation behind the use of chaotic UWB-MIMO waveforms for the proposed cognitive Bayesian DPMM-CP framework is to allow larger degrees of freedom in the selection of $T, T_{p}$, phase and amplitude over individual radar pulses which has a significant influence over the radar ambiguity function or, in other words, the rangeDoppler response offered by the UWB-MIMO radar. 


\section{DPMM CLUSTERING MECHANISM FOR MULTIPLE UAV TARGETS}

As shown in Fig. 1, the MIMO receiver unit isolates the orthogonal channel contributions by the UWB filter bank within the RF frontend. Subsequently, this filtered signal is passed to the matched filter for a particular MIMO channel $n$ where the range-Doppler estimates are generated. The same signal is processed to estimate the corresponding azimuth and elevation data by utilizing the 2D MUSIC algorithm. The aggregate mixture data represents a mixture of multivariate distribution classes over amplitude-azimuth-elevation, $\{\Gamma, \phi, \theta\}$. The DPMM clustering mechanism is invoked at this stage to discriminate between the underlying distributions over distinct UAV targets from the aggregate mixture over $\{\Gamma, \phi, \theta\}$ for a particular channel. This step is followed by indexing and assigning labels to clusters for channel $n$ and the corresponding range-Doppler estimates for each cluster along with the discriminated clusters is forwarded to the GLRT based detection module, subsequently followed by the $\mathrm{CP}$ algorithm and cognitive waveform design.

\section{A. DPMM Clustering Mechanism}

For a particular channel data, we assume that $\mathbf{s}_{n}$ follows a multivariate Gaussian distribution over the amplitude-azimuthelevation with mean vector $\mu_{i}$ and covariance matrix $\sigma_{i}$. Let $\psi_{i}=\left\{\mu_{i}, \sigma_{i}\right\}$ be the parameter of interest for data the $\mathbf{s}_{n}$. In order to discriminate between distinct extended targets, our goal is to find the posterior distribution of $\left(\psi_{1}, \cdots, \psi_{\kappa}\right)$ given the data, $\left(\mathbf{s}_{1}, \cdots, \mathbf{s}_{\kappa}\right)$. This posterior distribution will indicate the underlying multivariate distribution over each of the component target contributions. We develop the DPMM formulation as shown in [17], [25], [35], [36]. Suppose we make a sequence of observations $\mathbf{s}_{1}, \ldots, \mathbf{s}_{\kappa}$, where for each $i=1, \ldots, \kappa, \mathbf{s}_{i} \sim F\left(\cdot \mid \psi_{i}\right)$, and $\psi_{i} \in \Psi$ represents a parameter describing the observation distribution. In the Bayesian approach, we impose a prior distribution on $\left(\psi_{1}, \ldots, \psi_{\kappa}\right)$. In the DPMM, this prior is chosen to be a stochastic process, which leads to a model with very rich features. Specifically, the Dirichlet Process (DP) is a distribution over the space of all probability measures on $\Psi$. A random distribution $G$ on $\Psi$ is then drawn from this distribution, and given $G$, the parameters $\psi_{i}, i=1, \ldots, \kappa$, are independent and identically distributed according to $G$. To define the DP, we first let $G_{0}$ be a probability distribution over $\Psi$, which represents our prior belief about a parameter, and $\varrho$ be a positive number that serves as a weight between our prior belief and the information inferred from observed data. We say that $G$ is distributed as a DP, denoted as $G \sim \operatorname{DP}\left(\varrho, G_{0}\right)$, if for any finite measurable partition $\chi_{1}, \ldots, \chi_{r}$ of $\Psi$, we have

$$
\left(G\left(\chi_{1}\right), \ldots, G\left(\chi_{r}\right)\right) \sim \operatorname{Dir}\left(\varrho G_{0}\left(\chi_{1}\right), \ldots, \varrho G_{0}\left(\chi_{r}\right)\right)
$$

where $\operatorname{Dir}(\cdot)$ is the Dirichlet distribution. From this definition, we see that the DP is a stochastic process. Thus, the DPMM has the following representation

$$
\begin{aligned}
G & \sim \operatorname{DP}\left(\varrho, G_{0}\right), \\
\psi_{i} \mid G & \sim G, \\
\mathbf{s}_{i} \mid \psi_{i} & \sim F\left(\cdot \mid \psi_{i}\right) .
\end{aligned}
$$

Let $\psi_{-i}=\left(\psi_{1}, \ldots, \psi_{i-1}, \psi_{i+1}, \ldots, \psi_{\kappa}\right)$ be the vector of parameters excluding $\psi_{i}$. In the following, we assume that all distributions have a density with respect to some dominating $\sigma$-finite measure. Moreover, we will abuse notations and use the same symbols to denote the distribution as well as the density. The posterior distribution of $\psi_{i}$, conditioned on the data $s$ and $\psi_{-i}$ is then given by

$$
p\left(\psi_{i} \mid \psi_{-i}, \mathbf{s}_{i}\right) \propto F\left(\mathbf{s}_{i} \mid \psi_{i}\right) p\left(\psi_{i} \mid \psi_{-i}\right),
$$

since, given $\psi_{-i}, \psi_{i}$ depends only on $\mathbf{s}_{i}$. From the BlackwellMacQueen Polya-Urn scheme [35], the conditional distribution of $\psi_{i}$ given $\psi_{-i}$ is

$$
\begin{array}{r}
p\left(\psi_{i} \mid \psi_{-i}\right)=\frac{\varrho}{\varrho+n-1} G_{0}\left(\psi_{i}\right) \\
+\frac{1}{\varrho+n-1} \sum_{j \neq i} \delta_{\psi_{j}}\left(\psi_{i}\right),
\end{array}
$$

where $\delta_{\psi}$ is the Dirac delta function at $\psi$. Thus the posterior distribution (5) is given by

$$
\begin{array}{r}
p\left(\psi_{i} \mid \psi_{-i}, \mathbf{s}_{i}\right)=\varsigma \varrho G_{0}\left(\psi_{i}\right) F\left(\mathbf{s}_{i} \mid \psi_{i}\right) \\
+\varsigma \sum_{j \neq i} F\left(\mathbf{s}_{i} \mid \psi_{j}\right) \delta_{\psi_{j}}\left(\psi_{i}\right),
\end{array}
$$

where $\varsigma=1 /\left(\varrho q_{0}+\sum_{j \neq i} F\left(\mathbf{s}_{i} \mid \psi_{j}\right)\right)$ is a normalizing constant, and

$$
q_{0}=\int G_{0}(\psi) F\left(\mathbf{s}_{i} \mid \psi\right) \mathrm{d} \psi,
$$

is the marginal density of $\mathbf{s}_{i}$ at its realization. In order to evaluate the integral (8), we choose $G_{0}$ to be a conjugate prior to the Gaussian distribution $F\left(\mathbf{s}_{i} \mid \psi_{i}\right)$. In this work, the Normal-Wishart distribution for $G_{0}$ is used. A Gibbs sampler can now be designed to obtain the posterior distribution of $\psi_{i}$ given all the data as shown in [25]. Let $p_{s}\left(\psi_{i} \mid \psi_{-i}\right)$ be the conditional distribution of $\psi_{i}$ given all the data $s$. From (7), we sample $\psi_{i}$ according to

$$
p_{s}\left(\psi \mid \psi_{-i}\right)= \begin{cases}\varsigma F\left(\mathbf{s}_{i} \mid \psi_{j}\right), & \text { if } \psi=\psi_{j}, \\ \varsigma \varrho q_{0} \xi\left(\psi \mid \mathbf{s}_{i}\right), & \text { if } \psi \neq \psi_{j}, \forall j,\end{cases}
$$

where $\xi\left(\psi \mid \mathbf{s}_{i}\right)=G_{0}(\psi) F\left(\mathbf{s}_{i} \mid \psi_{i}\right) / q_{0}$.

We initialize the Gibbs sampler by considering each data $\mathbf{s}_{i}$ as being in its own set, with $\psi_{i}^{(0)}=\mathbf{s}_{i}$. Subsequently the Gibbs sampling for the $v$ th step is done in the following way.

- Sample $\psi_{1}^{v}$ from $p_{s}\left(\cdot \mid \psi_{2}=\psi_{2}^{(v-1)}, \psi_{3}=\psi_{3}^{(v-1)}, \ldots, \psi_{\kappa}=\right.$ $\left.\psi_{\kappa}^{(v-1)}\right)$

- Sample $\psi_{2}^{v}$ from $p_{s}\left(\cdot \mid \psi_{1}=\psi_{1}^{(v)}, \psi_{3}=\psi_{3}^{(v-1)}, \ldots, \psi_{\kappa}=\right.$ $\left.\psi_{\kappa}^{(v-1)}\right)$

- $\cdots$

- Sample $\psi_{\kappa}^{v}$ from $p_{s}\left(\cdot \mid \psi_{1}=\psi_{1}^{(v)}, \psi_{2}=\psi_{2}^{(v)}, \ldots, \psi_{\kappa-1}=\right.$ $\left.\psi_{\kappa-1}^{(v)}\right)$

The conventional GLRT based target detection approach determines the presence of the target in each range resolution 


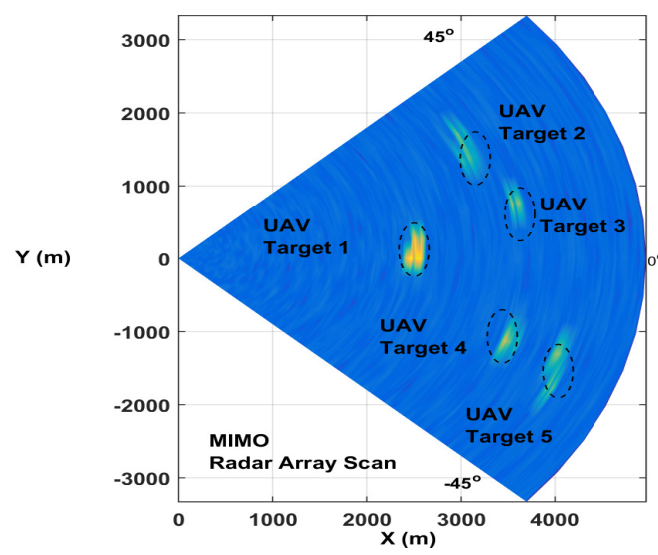

(a)

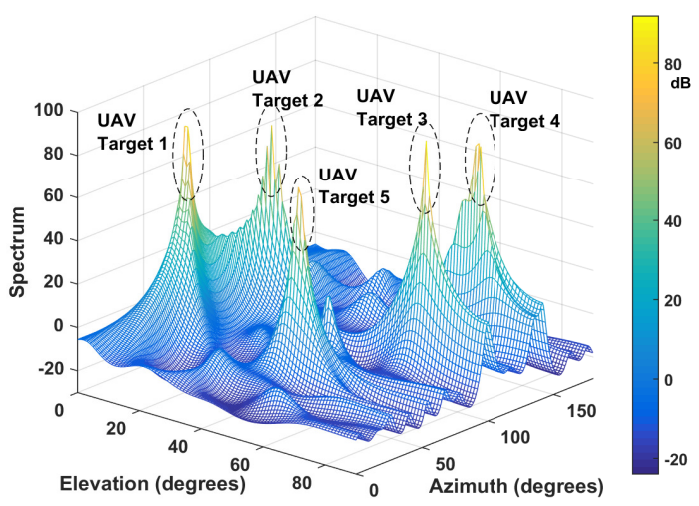

(b)

Fig. 2. (a) Range map extracted for 5 UAV targets from matched filter output for a single channel chaotic UWB waveform, (b) 2 D MUSIC algorithm based AOA estimation for 5 UAV targets.

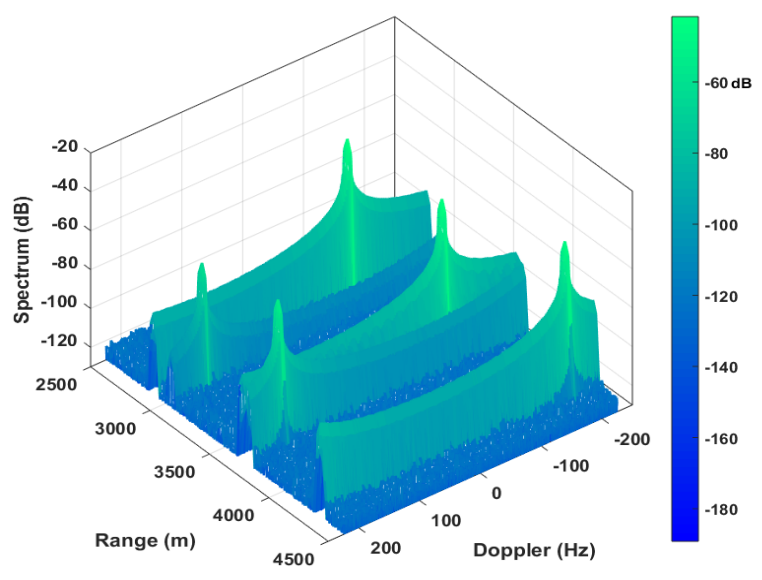

(a)

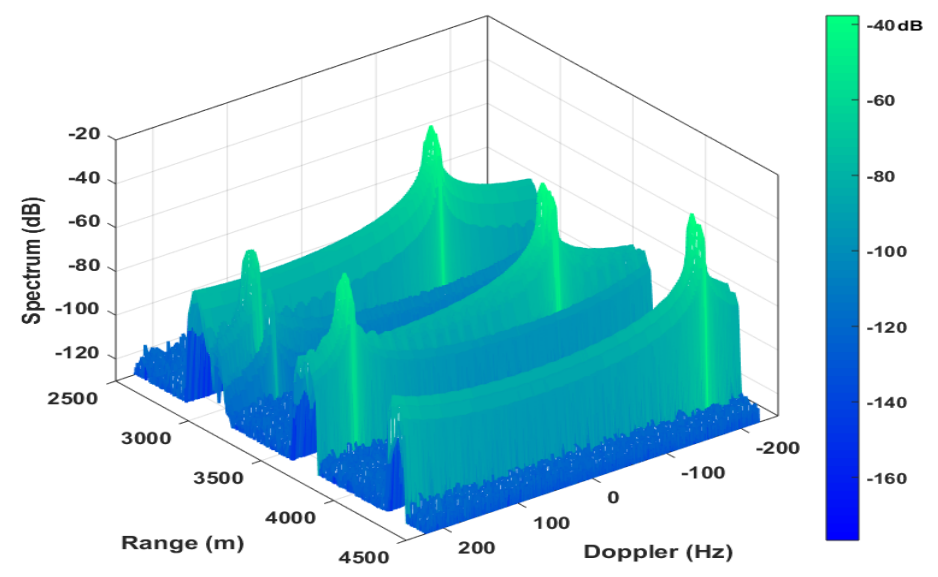

(b)

Fig. 3. Range-Doppler resolution for 5 UAV targets, (a) conventional fixed UWB waveform, (b) proposed chaotic UWB waveform.

cell. The proposed DPMM aided GLRT implements the GLRT detection mechanism on the discriminated clusters only, thus avoiding testing of GLRT test-statistic over each range and Doppler cell by modelling an unwieldy clutter covariance matrix. The GLRT maximizes the likelihood ratio test over the unknown parameters of interest like, $\zeta_{n}$ and $\rho_{n}$, where $\rho_{n}$ is the Doppler shift corresponding to MIMO channel $n$ due to unknown velocities of the target in $x, y$ and $z$ directions. We adopt the GLRT based detection as formulated in [37] over the DPMM clusters.

\section{Change Point Detection based DAA Radar}

\section{A. Model Representation}

Our objective in this section is to detect changes in the trajectories of the detected and discriminated UAV targets. The proposed change-point algorithm is applied to the KF estimates for the UAV target's $\{x, y, z, \theta, \phi\}$ parameters. Let $\mathbf{A}(i: j)=(A(i), A(i+1), \cdots, A(j))$ be a segment of the estimates from transmission frames $i$ to $j$. Suppose that A $(1: T)$ can be divided into $m$ segments, separated by the change points $\delta_{0}, \delta_{1}, \ldots, \delta_{m}$ with $\delta_{0}=0$ and $\delta_{m}=T$. For each segment $\mathbf{A}\left(\left(\delta_{i}+1\right): \delta_{i+1}\right), i=0, \ldots, m-1$, i.e., conditioned on the target parameter variation within a segment, we assume a linear regression model with order $l_{i}$ given by

$$
\mathbf{A}\left(\left(\delta_{i}+1\right): \delta_{i+1}\right)=\mathbf{G}_{i}^{\left(l_{i}\right)} C_{i}+\epsilon\left(\left(\delta_{i}+1\right): \delta_{i+1}\right),
$$

where $\mathbf{G}_{i}^{\left(l_{i}\right)}$ is a matrix of basis vectors, $C_{i}$ is a vector of parameters, and $\epsilon\left(\left(\delta_{i}+1\right): \delta_{i+1}\right)$ is a vector of independent and identically distributed random variables with mean 0 and the variance $\omega_{i}^{2}$. Our goal is to obtain the maximum a posteriori (MAP) estimates of the parameters $m$, and $\left\{\delta_{i}\right.$ : $i=1, \ldots, m-1\}$.

\section{B. Perfect Simulation}

The model in (10) has no analytical form for the posterior distributions of the parameters that we are interested in. 


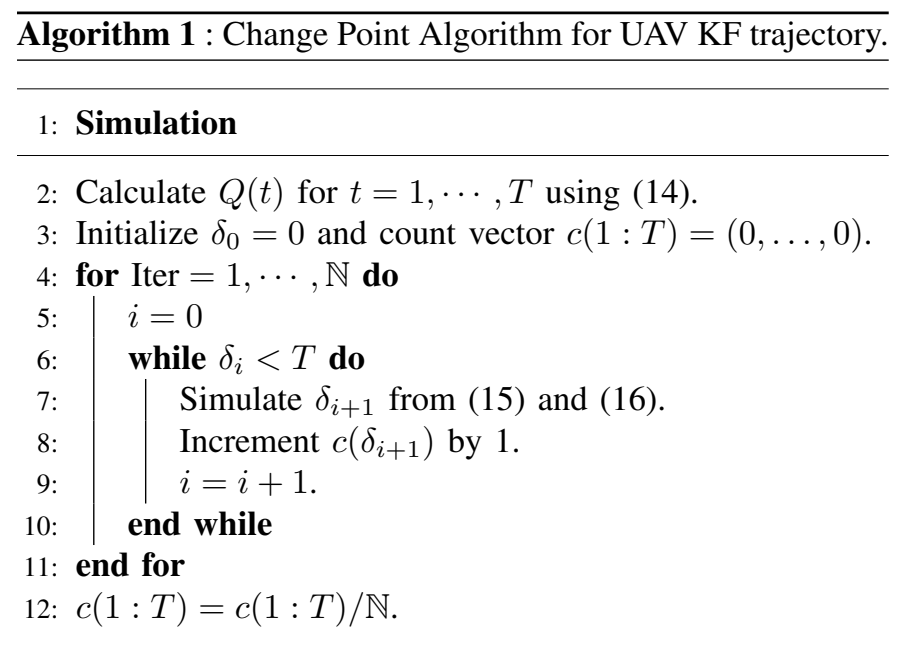

\section{3: Viterbi Algorithm}

14: Initialize $Q^{*}(T+1)=1$.

15: for $t=T, T-1, \ldots, 1$ do

16: $\mid Q^{*}(t)=\frac{1}{l} \max _{\substack{t \leq t^{\prime} \leq T \\ 1 \leq q \leq l}} \operatorname{Pr}\left(t, t^{\prime}, q\right) Q^{*}\left(t^{\prime}+1\right) \lambda^{\mathbf{1}\left(t^{\prime} \neq T\right)}(1-$ $\lambda)^{s-t}$

17: Set $t^{*}(t)$ and $q^{*}(t)$ to be the maximizers for $Q^{*}(t)$.

18: end for

19: Initialize $\delta_{0}^{*}=0$ and $j=0$.

20: while $\delta_{j}^{*}<T$ do

21: $\quad$ Set $\delta_{j+1}^{*}=t^{\prime *}\left(\delta_{j}^{*}+1\right)$ and $q_{j+1}^{*}=q^{*}\left(\delta_{j}^{*}+1\right)$.

22: $\quad j=j+1$.

23: end while

24: Number of change points $m=j$.

25: For each $\delta$ in $\left(\delta_{1}^{*}, \ldots, \delta_{m}^{*}\right)$, if there are other change points within $T$ second of $\delta$, keep only the change point with the highest $c(\delta)$. Update $m$ accordingly.

We therefore use Monte Carlo methods to perform Bayesian inference [38], [39]. The most common approach is the use of Markov chain Monte Carlo (MCMC) techniques. However, MCMC methods have the disadvantage of not being able to accurately determine if the procedure has converged, which may produce erroneous results [26]. In our setup, the observations in the disjoint segments are independent of each other; therefore we can adopt the so called perfect simulation approach of [26]-[28], which involves drawing independent samples from the true posterior distribution, and hence avoiding issues of convergence. In the following, we describe briefly the perfect simulation algorithm, and refer the reader to [26], [28] for details. We impose an Inverse-Gamma prior distribution $\mathbb{I} G$ with shape parameter $\nu / 2$ and scale parameter $\vartheta / 2$ on $\omega_{i}^{2}$, the variance of the noise variables in (10). For the $j$ th component in the regression parameter vector $C_{i}$, we use an independent normal distribution $\mathcal{N}\left(0, \omega_{i}^{2} \varepsilon_{j}^{2}\right)$ as the prior, where $\varepsilon_{j}$ is a fixed parameter. Furthermore, we assume that the model orders $l_{i}$ are bounded by a maximum order $l$, and we use a uniform prior for the model order of each segment. Since we have assumed that the UAV target parameters within every time frame are

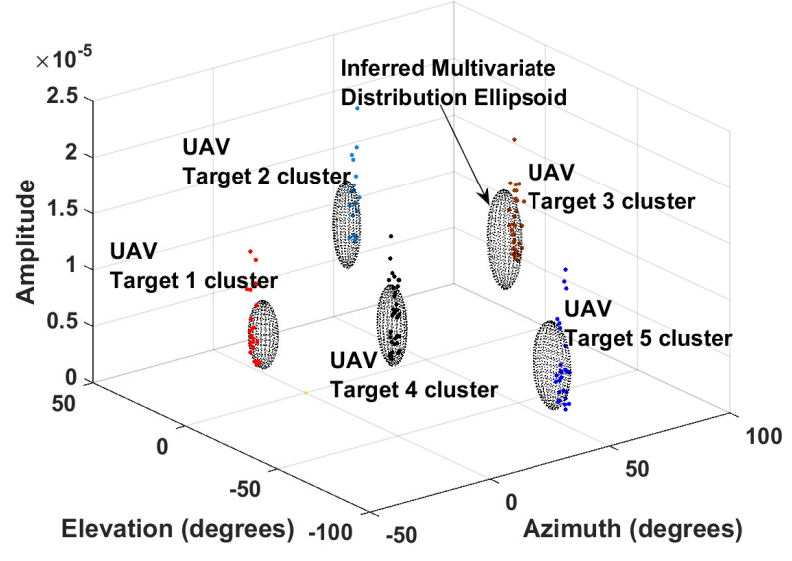

Fig. 4. Gibbs Sampling output for clustering the backscatter and inference on multivariate distributions over amplitude-elevation-azimuth for 5 targets.

independent, the prior on the change points is a geometric distribution, with the density function given by

$$
f\left(m, \delta_{1}, \cdots, \delta_{m-1}\right)=\lambda^{m-1}(1-\lambda)^{n-m},
$$

where $\lambda$ is a fixed parameter. The parameters $\left(\nu, \vartheta,\left(\varepsilon_{j}\right)_{j=1}^{2 l+1}, \lambda\right)$ can be chosen using a recursive procedure described in [26].

In the following, we present the necessary formulae that allow us to compute the posterior probability of a change point. We refer the reader to [26], [28] for additional details and derivations. Let $\operatorname{Pr}\left(t, t^{\prime}, q\right)$ be the conditional probability of the observations $\mathbf{A}\left(t: t^{\prime}\right)$, given that the model order is $q$. It can be shown that

$$
\begin{aligned}
\operatorname{Pr}\left(t, t^{\prime}, q\right) & =\operatorname{Pr}\left(\mathbf{A}\left(t: t^{\prime} \mid \text { segment order } q\right)\right) \\
& =\Upsilon^{1 / 2}\left(\nu+\|\mathbf{A}\|_{\mathbf{Q}}^{2}\right) \\
& \times \frac{\mathbb{I} \mathbb{G}\left(\frac{\vartheta+t^{\prime}-t+1}{2}\right)}{\mathbb{I} \mathbb{G}\left(\frac{\vartheta}{2}\right)} \prod_{j=1}^{2 q+1} \varepsilon_{j}^{-1},
\end{aligned}
$$

where $\Upsilon=\left(\mathbf{G}^{T} \mathbf{G}+\mathbf{D}^{-1}\right)^{-1}, \mathbf{Q}=\mathbf{I}-\mathbf{G} \Upsilon \mathbf{G}^{T},\|\mathbf{A}\|_{\mathbf{Q}}^{2}=$ $\mathbf{A}^{T} \mathbf{Q A}$, where $\mathbf{D}=\operatorname{diag}\left\{\varepsilon_{1}^{2}, \cdots, \varepsilon_{q}^{2}\right\}$ is the prior variance on the regression parameters for this segment and $\mathbf{I}$ be the identity matrix with dimensions $\left(t^{\prime}-t+1\right) \times\left(t^{\prime}-t+1\right)$. In this work, we define $\mathbf{G}$ as the basis vector matrix, assuming a piece-wise constant Auto-Regressive (AR) process. Thus, G can be defined as

$$
\mathbf{G}_{t: t^{\prime}}^{(l)}=\left(\begin{array}{cccc}
A_{t-1} & A_{t-2} & \cdots & A_{t-l} \\
A_{t} & A_{t-1} & \cdots & A_{t-l+1} \\
\cdots & \cdots & \cdots & \cdots \\
A_{t^{\prime}-1} & A_{t^{\prime}-2} & \cdots & A_{t^{\prime}-l}
\end{array}\right) .
$$

Let $Q(t)$ be the conditional distribution of observing $\mathbf{A}(t$ : $T$ ) given that there is a change point at $t-1$. This can be 


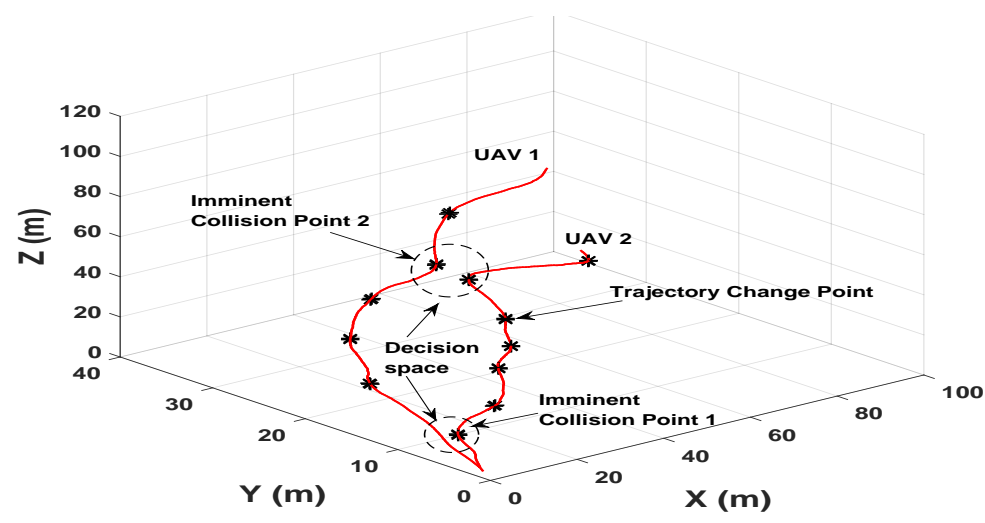

(a)

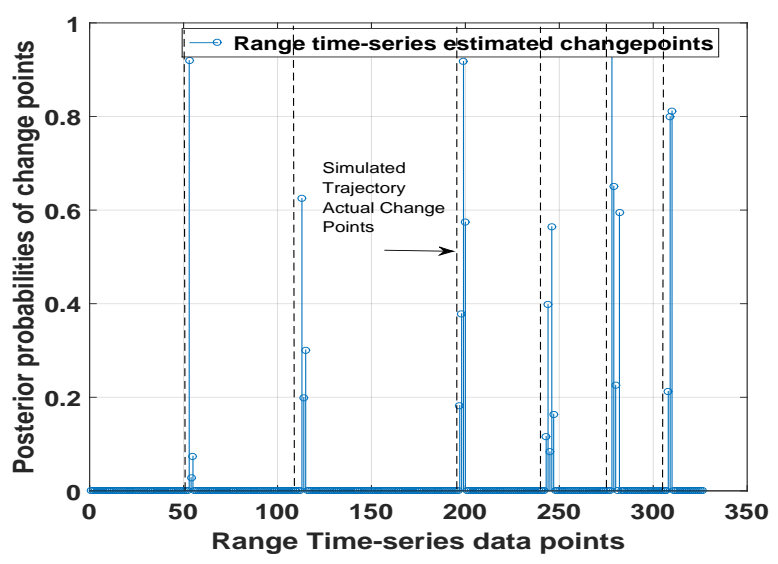

(b)

Fig. 5. Simulated UAV trajectories demonstrating the change-point based DAA mechanism, (a) UAV trajectories with imminent collision points, (b) perfect simulation for online determination of change points in range data time series.

calculated recursively using

$$
\begin{array}{r}
Q(t)=\frac{1}{l} \sum_{t^{\prime}=t}^{T-1} \sum_{q=1}^{l} \operatorname{Pr}\left(t, t^{\prime}, q\right) Q\left(t^{\prime}+1\right) \lambda(1-\lambda)^{t^{\prime}-t} \\
+\frac{1}{l} \sum_{q=1}^{l} \operatorname{Pr}(t, T, q)(1-\lambda)^{T-t} .
\end{array}
$$

The conditional probability of the next change point, given that the previous one occurred at $t-1$, is then given by

$$
\begin{array}{r}
\operatorname{Pr}\left(\delta_{j}=t^{\prime} \mid \delta_{j-1}=t-1, \mathbf{A}(1: T)\right) \\
\propto \frac{1}{l} \sum_{q=1}^{l} \operatorname{Pr}\left(t, t^{\prime}, q\right) Q\left(t^{\prime}+1\right) \lambda(1-\lambda)^{t^{\prime}-t} .
\end{array}
$$

and

$$
\begin{aligned}
\operatorname{Pr}\left(\delta_{j}\right. & \left.=T \mid \delta_{j-1}=t-1, \mathbf{A}(1: T)\right) \\
& \propto \frac{1}{l} \sum_{q=1}^{l} \operatorname{Pr}(t, T, q)(1-\lambda)^{T-t} .
\end{aligned}
$$

Making use of (15) and (16), we can simulate the next change point given the previous one until the last data point. This constitutes one run of the simulation process. We repeat this process several times and accumulate the count of the number of times that a particular point is determined to be a change point. We divide this count by the total number of runs and to obtain the posterior probability that this point is a change point. To find the MAP estimate of the change points, we use a Viterbi algorithm. This procedure is formally presented in the Algorithm 1.

\section{Simulation Results}

\section{A. Simulation parameters}

In this section, we present the simulation results for the proposed cognitive chaotic UWB-MIMO radar mechanism to facilitate autonomous UAV DAA navigation, as shown in Fig. 1. An extended target radar scenario comprising of

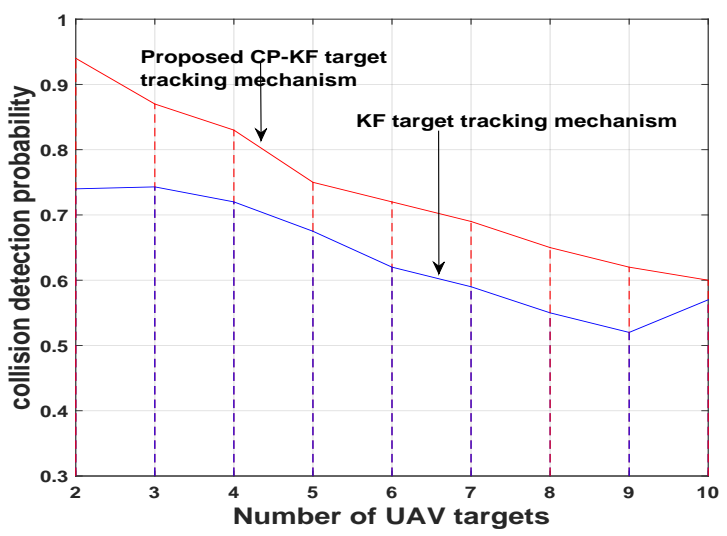

Fig. 6. Collision detection performance for the proposed CP-KF tracking mechanism.

Swerling II targets is simulated with a random number of scattering centers for distinct UAV targets. The chaotic UWBMIMO waveform uses one or more lengthy pseudo-random sequences to generate variation in phase, amplitude and PRI for the individual UWB monocycles described by (1). A large collection of such UWB waveforms represents the ensemble of such waveforms, which is to be used for selecting the waveform for transmission in the next instant. Such initiation of chaos based signals is perfectly consistent with other works on chaos based radar design [16].

Specifically in our work, we determine the values of $T, T_{p}$ identified by the DPMM-CP algorithm for a specific target of interest and then subsequently introduce chaotic variation within the UWB monocycles, as described by (1). 


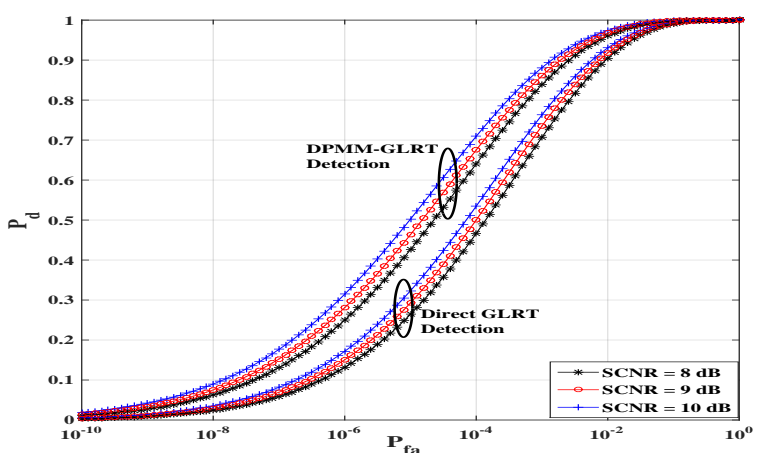

(a)

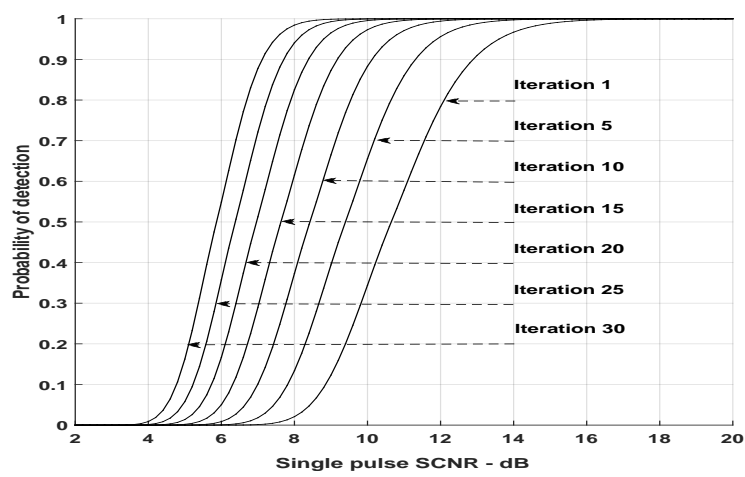

(b)

Fig. 7. (a) Receiver Operating Characteristics comparison for direct GLRT and DPMM assisted GLRT based detection strategy, (b) Variation in the probability of detection for the target of interest with the cognitive strategy.

\section{B. Chaotic UWB-MIMO radar range-Doppler and angular resolution}

As shown in Fig. 1, the UWB-MIMO 2D collects the captured signal within the angular space $\{\Theta, \Phi\}$. The match filtering operation for a particular channel and a beam-scan illustrated in Fig. 2(a). This plot displays the output of the matched filter for a particular channel for 5 UAV scenarios with extended targets. The chaotic variations in PRI, pulse width and amplitude enhance the detection of the individual scattering centers for each UAV target. In addition to the matched filtering operation, the captured backscatter is also fed to the 2D MUSIC algorithm in order to determine the azimuth and elevation angle estimates. Fig. 2(b) represents the angle estimation for a backscatter signal which is reflected from 3 and 5 UAV extended targets, respectively. As seen from this plot the individual UAV targets can be discriminated on the basis of their corresponding azimuth and elevation angle estimates. The DPMM assisted GLRT based detection is now applied to the output of the matched filter and corresponding AOA estimates, as shown in Fig. 1.

A significant advantage of the proposed chaos-based UWBMIMO signals is their enhanced target signature detection capability due to the chaotic variation in their PRI, pulse width, amplitude and phase, as described by (1). This advantage can be seen from the Range-Doppler resolution achieved by the matched-filter output shown in Fig. 3. Fig. 3(a) illustrates the range-Doppler resolution achieved by conventional UWBMIMO waveform wherein the PRI, pulsewidth, amplitude and phase are fixed. At the same time, Fig. 3(b) represents the range-Doppler resolution achieved by the proposed chaos based UWB-MIMO waveform. It can be seen from this result that the chaos based waveform design can reveal a larger number of target scattering centres over the 5 UAV targets than the conventional UWB-MIMO waveform design. This ability to reveal larger scattering centers over each extended UAV target facilitates enhanced target detection.

\section{DPMM clustering engine}

Upon the matched filtering and AOA estimation of the aggregate backscatter from the radar scene, the captured signal for a particular orthogonal channel is passed to the DPMM clustering, where the underlying multivariate distributions over $\{\Gamma, \phi, \theta\}$ within the received signal are inferred. This is achieved by the collapsed Gibbs sampling shown in Section III-A. These clustering results are shown in Fig. 4. The data points represent a mixture over amplitude-azimuth-elevation points for each scatterer return and the ellipsoids represent the inferred multivariate distribution over the data points.

\section{CP Algorithm for DAA Mechanism}

Fig. 5(a) shows the DAA mechanism for collision avoidance based on the proposed change point algorithm. The estimated location from the DPMM-GLRT based detection is passed to a standard KF tracker to track the trajectory for the target of interest. Based on the range estimates for the UAV for the target of interest, the perfect simulation algorithm described in Section IV-B is implemented as shown in Algorithm 1.

Fig. 5(b) represents the estimated change points derived from the posterior distribution over the range time-series; this is used to infer the sudden changes in the trajectory of the UAV target. Fig. 5(a), illustrates the DAA strategy implemented by UAV 2 based on UAV 1 estimated range time-series data points. As seen from this figure two imminent collision instances are averted autonomously by UAV 2 thanks to the coarse correction enabled by the estimation on change points shown in Fig. 5(b).

Fig. 6 represents the performance improvement within collision detection presented by the CP-KF tracking mechanism. Specifically, we simulate 100 individual trajectories for each of the 10 UAV targets similar to the ones shown in Fig. 5 (a) within a confined $3 D$ space of $2 \mathrm{~km} \times 2 \mathrm{~km} \times 2 \mathrm{~km}$. The average hypersonic UAV drone velocity is assumed to be Mach 1. Based upon this average velocity and simulated UAV tracks we determine the total number of imminent collision points (time to collision $<30 \mathrm{sec}$ ) and also determine the successful collision detection points calculated by the CPKF algorithm. Subsequently, we compute the probability of collision detection through the proposed $\mathrm{CP}-\mathrm{KF}$ mechanism and through a more conventional KF tracking scheme. As demonstrated by the result in Fig. 6, the proposed CP-KF 
mechanism outperforms the conventional KF tracker based approach significantly in estimation of imminent collision points. This performance improvement can be attributed to the fact that the $\mathrm{CP}-\mathrm{KF}$ algorithm refines the collision detection estimation by computing the posterior distribution over the trajectory change points as shown in Fig. 5(b).

\section{E. Advantage of DPMM assisted GLRT and overall cognitive DAA strategy}

Fig. 7(a) represents the Receiver Operating Characteristic (ROC) curves for the proposed cognitive approach over 3 distinct SCNR floors for direct GLRT based detection and the proposed DPMM-GLRT based detection. The ROC curves are generated by averaging over a 1000 realizations of the received backscatter signal at a fixed SCNR values of $8 \mathrm{~dB}-10 \mathrm{~dB}$. The area under the ROC curves indicates the superior performance of the proposed DPMM-GLRT based detection approach. Fig. 7(b) displays the variation in the probability of UAV target detection with varying iteration count over chaotic UWB waveform selection. In particular, for each iteration the values of $T_{p}, T$ and UWB monocycle amplitudes within the chaotic UWB-MIMO waveform have been modified for the identified target of interest. The result in Fig. 7(b) demonstrates this enhanced probability of target detection due to the cognitive selection of these parameters.

\section{CONCLUSion AND Future Works}

We have demonstrated the application of UWB-MIMO radar for DAA mechanism in order to facilitate autonomous UAV navigation. Chaos based UWB-MIMO waveforms offer superior flexibility in range-Doppler resolution for the collection of individual scatterers within the radar scene. The proposed DPMM-CP based algorithm not only provides an unsupervised mixture component analysis mechanism to discriminate distinct UAV target scatterers without making any a priori target scene assumptions but also facilitates the online detection of change points in the trajectory of the UAV targets in the vicinity and thus provides vital assistance to the guidance and navigation control of the UAV system to adapt its course and avoid imminent collisions autonomously. The overall chaotic UWB-MIMO radar parameters can be adapted on the basis of current location and velocity estimates for the target of interest, thus giving rise to the cognitive mechanism which significantly enhances the UAV target detection probability. Future works could be focused upon the development of passive radar architectures which exploit the signals of opportunity such as DVB-T/ATSC, FM radio and cellular transmissions, etc. for enabling DAA mechanisms for UAVs. This would considerably lower the on-board transmission power and cost requirements. Moreover, software-definedradio based transceivers could also be employed onboard UAVs to facilitate cognitive waveform design and practical realization for the proposed DAA approach.

\section{REFERENCES}

[1] S. Rathinam, P. Almeida, Z. Kim, S. Jackson, A. Tinka, W. Grossman, and R. Sengupta, "Autonomous Searching and Tracking of a River using an UAV," in Proceedings of American Control Conference, ACC '07, Jul. 2007, pp. 359-364.
[2] Z. Li and J. Ding, "Ground Moving Target Tracking Control System design for UAV Surveillance," in Proceedings of IEEE International Conference on Automation and Logistics, Aug. 2007, pp. 1458-1463.

[3] E. Semsch, M. Jakob, D. Pavlicek, and M. Pechoucek, "Autonomous UAV Surveillance in Complex Urban Environments," in Proceedings of IEEE/WIC/ACM International Joint Conferences on Web Intelligence and Intelligent Agent Technologies, vol. 2, Sept. 2009, pp. 82-85.

[4] P. Olsson, J. Kvarnstrom, P. Doherty, O. Burdakov, and K. Holmberg, "Generating UAV communication networks for monitoring and surveillance," in Proceedings of 11th International Conference on Control Automation Robotics Vision (ICARCV), Dec. 2010, pp. 1070-1077.

[5] M. Bhaskaranand and J. Gibson, "Low Complexity Video Encoding and High Complexity Decoding for UAV Reconnaissance and Surveillance," in Proceedings of IEEE International Symposium on Multimedia (ISM), Dec. 2013, pp. 163-170.

[6] E. George, G. Tiwari, R. Yadav, E. Peters, and S. Sadana, "UAV systems for parameter identification in agriculture," in Proceedings of IEEE Global Humanitarian Technology Conference: South Asia Satellite (GHTC-SAS), Aug. 2013, pp. 270-273.

[7] F. A. Administration, "Press release DOT and FAA Propose New Rules for Small Unmanned Aircraft Systems," Washington, D.C., Feb. 2015.

[8] T. Zsedrovits, P. Bauer, A. Zarandy, B. Vanek, J. Bokor, and T. Roska, "Error analysis of algorithms for camera rotation calculation in gps/imu/camera fusion for uav sense and avoid systems," in International Conference on Unmanned Aircraft Systems (ICUAS), May 2014, pp. 864-875.

[9] Z. Ma, T. Hu, L. Shen, W. Kong, and B. Zhao, "A detection and relative direction estimation method for UAV in sense-and-avoid," in IEEE International Conference on Information and Automation, Aug 2015, pp. 2677-2682.

[10] R. Kephart and M. Braasch, "See-and-avoid comparison of performance in manned and remotely piloted aircraft," IEEE Aerospace and Electronic Systems Magazine, vol. 25, no. 5, pp. 36-42, May 2010.

[11] D. Accardo, G. Fasano, L. Forlenza, A. Moccia, and A. Rispoli, "Flight Test of a Radar-Based Tracking System for UAS Sense and Avoid," IEEE Transactions on Aerospace and Electronic Systems, vol. 49, no. 2, pp. 1139-1160, Apr. 2013.

[12] R. Holdsworth, J. Lambert, and N. Harle, "Inflight Path planning replacing pure collision avoidance, using ADS-B," IEEE Aerospace and Electronic Systems Magazine, vol. 16, no. 2, pp. 27-32, Feb. 2001.

[13] Y. Zhang and G. Fairley, "Multiple-Trajectory-Prediction (MTP) algorithm for UAS's Sense and Avoid (SAA) operation," in Proceedings of Integrated Communications, Navigation and Surveillance Conference (ICNS), 2013, Apr. 2013, pp. 1-11.

[14] B. Stark, B. Stevenson, and Y. Chen, "ADS-B for small Unmanned Aerial Systems: Case study and regulatory practices," in Proceedings of International Conference on Unmanned Aircraft Systems (ICUAS), 2013, May 2013, pp. 152-159.

[15] M. Strohmeier, M. Schafer, V. Lenders, and I. Martinovic, "Realities and challenges of nextgen air traffic management: the case of ADS-B," IEEE Communications Magazine, vol. 52, no. 5, pp. 111-118, May 2014.

[16] V. Venkatasubramanian, H. Leung, and X. Liu, "Chaos UWB Radar for Through-the-Wall Imaging," IEEE Transactions on Image Processing, vol. 18, no. 6, pp. 1255-1265, Jun. 2009.

[17] Y. Nijsure, G. Kaddoum, and H. Leung, "Cognitive chaotic UWBMIMO radar based on nonparametric Bayesian technique," IEEE Transactions on Aerospace and Electronic Systems, vol. 51, no. 3, pp. 23602378, July 2015.

[18] T. Naghibi and F. Behnia, "MIMO Radar Waveform Design in the presence of clutter," IEEE Transactions on Aerospace and Electronic Systems, vol. 47, no. 2, pp. 770-781, Apr. 2011.

[19] G. Tzortzis and C. Likas, "The Global Kernel k -Means Algorithm for Clustering in Feature Space," IEEE Transactions on Neural Networks, vol. 20, no. 7, pp. 1181-1194, Jul. 2009.

[20] E. Fox, D. Choi, and A. Willsky, "Nonparametric Bayesian Methods for Large Scale Multi-Target Tracking," in Proceedings of Fortieth Asilomar Conference on Signals, Systems and Computers, Nov. 2006, pp. 2009 -2013 .

[21] N. Bouguila and D. Ziou, "High-Dimensional Unsupervised Selection and Estimation of a Finite Generalized Dirichlet Mixture Model Based on Minimum Message Length," IEEE Transactions on Pattern Analysis and Machine Intelligence, , vol. 29, no. 10, pp. 1716 -1731, Oct. 2007.

[22] E. Jackson, M. Davy, A. Doucet, and W. Fitzgerald, "Bayesian Unsupervised Signal Classification by Dirichlet Process Mixtures of Gaussian Processes," in Proceedings of IEEE International Conference on Acoustics, Speech and Signal Processing, vol. 3, Apr. 2007, pp. 1077-1080. 
[23] L. Sun, Y. Zhang, G. Tian, and M. Ma, "SAR Image Segmentation Using GHM-Based Dirichlet Process Mixture Models," in Proceedings of International Joint Conference on Computational Sciences and Optimization, vol. 1, Apr. 2009, pp. 886 -888.

[24] Y. Nijsure, W. P. Tay, E. Gunawan, and L. C. Joshua, "A Bayesian Nonparametric Approach to Tumor Detection Using UWB Imaging," in Proceedings of IEEE International Conference on Ultra-Wideband (ICUWB), Sept. 2012.

[25] R. Neal, "Markov chain sampling methods for Dirichlet process mixture models." Journal of Computational and Graphical Statistics, vol. 9, pp. 249-265, 2000.

[26] Fearnhead, P., "Exact Bayesian curve fitting and signal segmentation," IEEE Transactions on Signal Processing, vol. 53, no. 6, pp. 2160-2166, Jun. 2005.

[27] Fearnhead, P., "Exact and efficient Bayesian inference for multiple changepoint problems," Statistics and Computing, vol. 16, pp. 203-213, 2006.

[28] Y. Nijsure, W. P. Tay, E. Gunawan, F. Wen, Z. Yang, Y. L. Guan, and A. P. Chua, "An Impulse Radio Ultrawideband System for Contactless Noninvasive Respiratory Monitoring," IEEE Transactions on Biomedical Engineering, vol. 60, no. 6, pp. 1509-1517, June 2013.

[29] A. Moses, M. Rutherford, and K. Valavanis, "Radar-based detection and identification for miniature air vehicles," in IEEE International Conference on Control Applications (CCA), Sept. 2011, pp. 933-940.

[30] L. Shi, C. Allen, M. Ewing, S. Keshmiri, M. Zakharov, F. Florencio, N. Niakan, and R. Knight, "Multichannel sense-and-avoid radar for small UAVs," in IEEE/AIAA 32nd Digital Avionics Systems Conference (DASC), Oct. 2013, pp. 6E2-1-6E2-10.

[31] D. Klarer, P. Feil, and M. Edrich, "Design considerations of airborne sense and avoid radars," in 16th International Radar Symposium (IRS), June 2015, pp. 219-224.

[32] H.-C. Lee, "Implementation of collision avoidance system using TCAS II to UAVs," IEEE Aerospace and Electronic Systems Magazine, vol. 21, no. 7, pp. 8-13, July 2006.

[33] K. James and D. Ann, "The Traffic Alert and Collision Avoidance System," MIT Lincoln Laboratory Journal, vol. 16, no. 2, Jul. 2007.

[34] A. Hassanien, M. Morency, A. Khabbazibasmenj, S. Vorobyov, J.Y. Park, and S.-J. Kim, "Two-dimensional transmit beamforming for MIMO radar with sparse symmetric arrays," in Proceedings of IEEE Radar Conference (RADAR) 2013, Apr. 2013, pp. 1-6.

[35] D. Blackwell and J. MacQueen, "Ferguson distributions via polya urn schemes," Annals of Statistics, pp. 353-355, 1973.

[36] M. Escobar and M.West, "Bayesian density estimation and inference using mixtures," J. Am. Stat. assoc., vol. 90, pp. 577-588, 1995.

[37] A. M. Haimovich, R. S. Blum, and L. J. Cinimi, "MIMO radar with widely separated antennas," IEEE Signal Processing Magazine, vol. 25, no. 1, pp. 116-129, Jan. 2008.

[38] T. Y. Yang and L. Kuo, "Bayesian binary segmentation procedure for a Poisson process with multiple changepoints," Journal of Computational and Graphical Statistics, vol. 10, pp. 772-785, 2001.

[39] D. Barry and J. Hartigan, "A Bayesian analysis of change point problems," Applied Statistics, vol. 88, pp. 309-319, 1993.

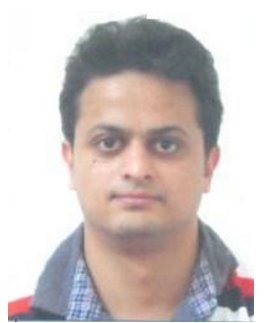

Yogesh Anil Nijsure received the B.E. degree (Distinction) in Electronics Engineering from University of Mumbai, India, in June 2006 and received his M.Sc. degree (Distinction, Rank 1) in Wireless Communication Systems Engineering from the University of Greenwich, U.K. in September 2008. $\mathrm{He}$ received his $\mathrm{Ph} . \mathrm{D}$. degree from the University of Newcastle upon Tyne in U.K. in October 2012. From March 2010 to September 2010 he undertook his research internship at the Institute for Infocomm Research (I2R), Singapore, as a research engineer. From November 2011 to November 2012 he worked as a Research Associate at Nanyang Technological University, Singapore. From December 2012 to April 2014, he undertook aerospace research at Rockwell Collins, India. Since April 2014, he has been working as a postdoctoral research fellow at the Ecole de technologie superieure (ETS), University of Quebec located in Montreal, Canada. His research interests include cognitive radar network design, Bayesian non-parametric methods, UWB radar systems, robust ADSB multilateration systems, cognitive radio networks, information theory, radar signal processing, electronic warfare and software defined radio systems.

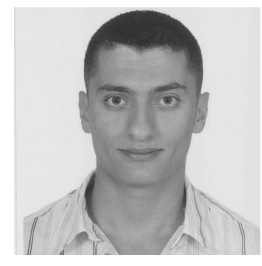

Georges Kaddoum received the Bachelors degree in electrical engineering from the Ecole Nationale $\mathrm{Su}$ prieure de Techniques Avances (ENSTA Bretagne), Brest, France; the M.S. degree in telecommunications and signal processing (circuits, systems, and signal processing) from the Universit de Bretagne Occidentale and Telecom (ENST) Bretagne, Brest, in 2005; and the Ph.D. degree (with honors) in signal processing and telecommunications from the National Institute of Applied Sciences (INSA), University of Toulouse, Toulouse, France, in 2009. He is an Assistant Professor of electrical engineering with the Ecole de Technologie Suprieure (ETS), University of Quebec, Montreal, QC, Canada. He was a Scientific Researcher with ETS in 2012 and was then promoted to Assistant Professor in November 2013. In 2014, he was awarded the ETS Research Chair in physical-layer security for wireless networks. Since 2010, he has been a Scientific Consultant in the field of space and wireless telecommunications for several companies (Intelcan Techno-systems, MDA Corporation, and Radio-IP companies). He has published over 60 journal and conference papers and has two pending patents. His recent research activities cover wireless communication systems, chaotic modulations, secure transmissions, and space communications and navigation. Dr. Kaddoum received the Best Paper Award at the IEEE International Conference on Wireless and Mobile Computing, Networking, and Communications (WiMob 2014) with three other coauthors; and the 2015 IEEE Transactions on Communications Top Reviewer Award.

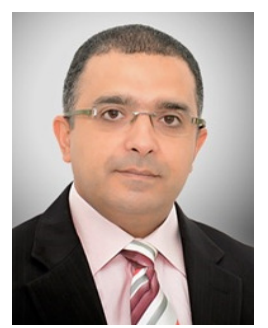

Nazih Khaddaj Mallat : (M'07-SM'12) received his Bachelor of Engineering degree (Electrical and Computer Engineering) from the Lebanese University in 2000, his Master degree from the "Ecole Nationale Suprieure des Telecommunications de Bretagne (ENSTB)", France, in 2002 and his Ph.D. degree in Telecommunication from University of Quebec, "Institut National de la Recherche Scientifique (INRS)", Canada, in 2010. After his Ph.D. and till January 2012, he was postdoctoral fellowship in Ecole Polytechnique de Montreal. The "Fonds Quebecois de la Recherche sur la Nature et les Technologies-FQRNT", a granting agency of the Quebec government, has awarded him two prestigious scholarships for his doctoral studies (2008) and postdoctoral research (20102011) thanks to his highest level of achievement. His main research interests are passive microwave/millimeter-wave circuit design, telecommunication systems. He authored or co-authored over 20 publications, mostly focused in multi-port applications, millimeter-wave circuits and telecommunications systems. His research results are presented at international conferences and submitted for journal publications. Over the last decade, Dr. Khaddaj Mallat has acquired extensive teaching experience at both undergraduate and graduate levels. He has effectively taught many courses, and their relevant practical elements in laboratories at multiple Montreal universities (ETS, TELUQ, Ecole Polytechnique de Montreal). Since 2006, he has been extremely involved with the IEEE Montreal Section. He was the vice-chair of the IEEE Montreal Section 2007-2008, Membership Development Chair in 2009-2010, and Section Chair in 2011-2012. He has been serving also in the steering committee of many IEEE international conferences: EPC2007, SMC2007, EPEC2009, CNSR2010, MWP2010, FBW2011, CCECE2012, and IMS2012. Dr. Khaddaj Mallat was elected in 2012 to become a member of the IEEE Canadian Foundation (ICF). In 2013, he joined the College of Engineering and Information Technology at Al Ain University of Science and Technology (AAU) in United Arab Emirates (UAE) as Assistant Professor. He has been the Head of Networks and Communication Engineering and Computer Engineering Department since 2013, Deputy Dean of the College of Engineering and Information Technology from April 2014 to August 2015, and Dean of the College of Engineering and Information Technology since September 2015. $\mathrm{He}$ is the founder of the IEEE AAU Student Branch and the IEEE UAE MTT$S$ Chapter. He is currently the IEEE UAE Technical Activities Coordinator and IEEE Region 8 Chapter Coordination Subcommittee (ChCSC) Chair. 


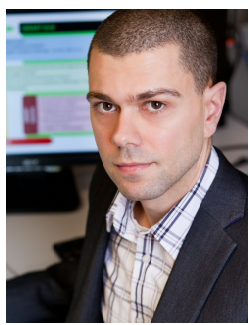

Ghyslain Gagnon received his B.Eng. and M.Eng. degrees in electrical engineering from the Ecole de technologie superieure, Montreal, Canada in 2002 and 2003 respectively. He also received the Ph.D. degree in electrical engineering from Carleton University, Canada in 2008. From 2003 to 2004, he worked for ISR Technologies, where he designed and implemented several critical synchronization modules for a software defined radio which later obtained the editors' choice award in 2007 by a portable design magazine. He is now an associate professor with the department of Electrical Engineering, Ecole de technologie superieure. He is inclined towards industrial research partnerships. His research aims at mixedsignal circuits and systems, as well as digital signal processing.

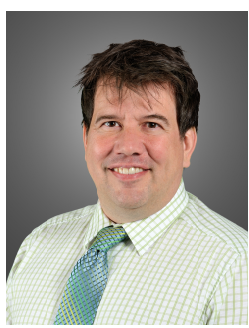

Francois Gagnon (S'87-M'87-SM'99) received his B.Eng. and $\mathrm{Ph} . \mathrm{D}$. degrees in electrical engineering from the Ecole Polytechnique de Montreal. Since 1991 he has been a professor with the Department of Electrical Engineering, Ecole de Technologie Superieure. He chaired the department from 1999 to 2001, and now holds the NSERC Ultra Electronics Chair, Wireless Emergency and Tactical Communication, at the same university. His research interests cover wireless high-speed communications, modulation, coding, high-speed DSP implementations, and military point-to-point communications. He has been very much involved in the creation of the new generation of high-capacity line-of-sight military radios offered by the Canadian Marconi Corporation, which is now Ultra Electronics Tactical Communications Systems. For its product, the company has received a "Coin of Excellence" from the U.S. Army for performance and reliability. He was awarded the 2008 NSERC Synergy Award (Small and Medium-Sized Companies category) for fruitful and long lasting collaboration with Ultra Electronics TCS. 